\title{
Eus-guided therapy for pancreatic pain: easy, effective and safe. why isn't the first therapeutic approach?
}

\begin{abstract}
Visceral pain is often a disabling symptom that appears in many chronic digestive diseases. In the case of pancreatic disease it's especially important, being most of the times the main symptom and interfering with nutrition, emotional state and life quality of these patients. The etiology of pancreatic pain is multifactorial, having neuropathic paths a main role. The current management of pancreatic pain follows the World Health Organization three-step ladder for pain control, but this classic model seems sometimes insufficient in pancreatic disease. Lately, therapeutic endoscopic ultrasound (EUS) has emerged as an effective alternative in pancreatic pain treatment, since the first descriptions of EUS-guided celiac plexus block and plexus neurolysis. Several studies confirm the effectiveness of these techniques, showing better results compared with those performed under fluoroscopy guidance, with less adverse effects and complications. In addition, they are easy and safe interventions, which can be performed by any endoscopist trained in basic therapeutic EUS. Both celiac plexus block (CPB) and celiac plexus neurolysis (CPN) are performed using a lineal therapeutic echoendoscope, injecting different substances in order to reduce the effects of celiac plexus activity. CPB is mainly recommended in pain due to chronic pancreatitis a well as CPN is preferred in pancreatic cancer, being more effective than $\mathrm{CPB}$ even with only one session. In this review we pretend to summarize the state-of-art in EUS-guided therapy, focusing in treatment of chronic pancreatic pain, neoplastic and benign, reviewing the main studies of effectiveness and comparing with other invasive techniques. In addition, we would like to evaluate the effectiveness of combined treatment, according to recent publications.
\end{abstract}

Keywords: Celiac plexus neurolysis, celiac plexus block, pancreatic adenocarcinoma, chronic pancreatitis, celiac ganglia, endoscopic ultrasonography
Special Issue - 2018

\author{
Pilar Castro Carbajo, ' Lydia Elena Pradera \\ Andrés, ${ }^{2}$ Carolina Navarro, ${ }^{3}$ Luis Ramón \\ Rábago Torres ${ }^{4}$ \\ 'De Aparato Digestivo, Hospital Universitario Severo Ochoa. \\ Avda. Orellana S/N 289/4, Leganés, Spain \\ ${ }^{2}$ S.A. R. Navalcarnero, Comunidad De, Spain \\ ${ }^{3}$ Hospital Universitario Ramón Y Cajal, Spain \\ ${ }^{4}$ Hospital Universitario Severo Ochoa, Spain
}

Correspondence: Pilar Castro Carbajo, Department Of Servicio De Aparato Digestivo, Hospital Universitario Severo Ochoa. Avda. Orellana S/N 289|4, Leganés, Spain, Tel +34654873691, Email Mpcastrocarbajo@Gmail.Com

Received: February 26, 2018| Published: September 19, 2018
Abbreviations: QOL, quality of life; WHO, world health organization; NSAID, no steroidal anti-inflammatory drugs; CPN, celiac plexus Neurolysis; CPB, celiac plexus block; CT, computerized tomography; EUS, endoscopic ultrasonography; FNA, fine needle aspiration; CGN, celiac ganglia Neurolysis; CGB, celiac ganglia block

\section{Introduction}

Visceral pain is a symptom that frequently appears in multiple digestive pathologies. In pancreatic diseases case it's especially relevant, since in many occasions it constitutes the main symptom, both in neoplastic diseases and benign pathology. It significantly affects the patient's quality of life (QOL) and requires high analgesic doses, frequently opioids, with its consequent side effects. The main pancreatic diseases in which pain constitutes a relevant symptom to be considered are pancreatic adenocarcinoma, especially in advanced cases, and chronic pancreatitis. Pancreatic pain has a multifactorial etiology, involving multiple neuropathic ways, as well as mechanical factors due to local neoplastic extension to adjacent organs, or due to parenchymal chronic changes (e.g. calcifications) in case of chronic pancreatitis. Pancreatic pain approach is initially based on the WHO's analgesic ladder, starting treatment at initial phases with non-opioid medication, mainly acetaminophen and non-steroid anti-inflammatory drugs (NSAIDs), and continuing with ascending doses of opioids until reaching pain control. This approach does not lack side effects, especially related to opioid treatment, which can limit doses' ascend and thus avoid a satisfactory pain control. For this reason it seems necessary to count on alternative therapies that allow lowering the drug's doses and achieving a better pain control in these pathologies.

\section{Celiac plexus neurolysis (CPN) and celiac plexus block (CPB)}

Celiac plexus ablation or blocking centered technics have been widely used since Kappis first described them more than 100 years ago. ${ }^{1}$ The celiac plexus is located caudal to the diaphragm, in an antecrural position, surrounding the celiac trunk base, and it comprises a ganglia and nervous fibers bundle which is interconnected. The number of ganglia and its size varies amongst subjects as well as its anatomic location in relation with the vertebral column, though it maintains a constant position adjacent to the celiac trunk. The celiac plexus transmits a painful signal originating from the pancreas and from most of abdominal viscera, except for left colon, rectum and pelvic viscera. For these reasons, the neuropathic interruption at this point can provide an important pain relief in multiple abdominal pathologies, especially neoplastic. It's important to differentiate CPN, in which a definitive destruction of the celiac plexus is aimed for, and $\mathrm{CPB}$ which provides a temporary block and pain relief via an anesthetic and corticoid injection. The efficiency of these technics has been evaluated in both pancreatic adenocarcinoma and chronic pancreatitis. Percutaneous approach has been the most frequently used, varying the access point location and the way to control the correct insertion of the needle, being possible to do it under fluoroscopic control, CT 
or even abdominal ultrasonography. In relation to the access, it can be anterior or posterior depending on the technic used. In published studies the posterior access with fluoroscopic control predominates, or recently with $\mathrm{CT} .^{2}$ There also exists the possibility to practice $\mathrm{CPN}$ with alcohol or other substances during surgical resection of the neoplasm or in a palliative surgery context. ${ }^{3}$ Such a technic would be an alternative to classic surgery, being its efficiency equivalent to the rest of approaches.

CPN's global efficiency has been proved in multiple studies, though quite heterogeneous in its designs. In a classic meta-analysis published by Eisenberg et al which includes a total of 24 studies, it's concluded that CPN achieves an abdominal oncological pain release (including various types of non-surgical neoplasms) of up to $90 \%$ after three months, and of $70 \%$ to $90 \%$ throughout the disease. ${ }^{4}$ It even suggests a longer life expectancy in patients in which this technic is applied. Other prospective and randomized studies have later confirmed these data ${ }^{5-7}$ although with more modest results, proving pain relief in most cases and a moderate descent in opioid doses and its side effects. A review published in 2007 concluded that although CPN's efficiency is unquestionable in relation to pain relief, it is not sufficiently efficient to be able to substitute conventional analgesic treatment, and it doesn't seem to improve the QOL or survival expectancy in oncologic patients. ${ }^{8}$ A recent retrospective study with a wide patient cohort finds that patients subject to CPN, in spite of the used technic, show a statistically significant reduction of survival rates. ${ }^{9}$ This surprising find contradicts previous studies. It is not clear if this translates an association between a more serious oncologic case and the tendency to use more invasive technics, or if the reduction of survival rates can be a direct cause of the technic. Since currently it's the only paper that reflects these results, prospective studies will be necessary to confirm them.

The most frequent complications of $\mathrm{CPN}$ are orthostatic hypotension and diarrhea, both of which appear in up to $40 \%$ of the patients. Pain exacerbation in the days following the injection is also frequent and self-limited. In posterior approach technics $2 \%$ of neurologic complications have been notified, including paraplegia, and $1 \%$ of pneumothorax, secondary to supra-diaphragmatic puncture. ${ }^{4}$ Anterior approach seems to avoid these severe complications according to currently available data. There exist few publications in which CPN use in benign pathology or in resectable cancer is evaluated. Lavu et al. have recently evaluated intra-surgical CPN efficacy in resectable peri-ampular and pancreatic adenocarcinomas. In this randomized prospective study with a total of 467 patients, it was concluded that there is no significant improvement in QOL or pain control during post-surgery. An increase in patient survival wasn't recorded either. It is also believed that the pre-surgical alcohol injection in the pancreatic area can stimulate fibrosis, making resection of the neoplasm of that location more difficult. In chronic pancreatitis it is believed that it can even worsen the pain and accelerate the disease in some cases. In spite of this some studies have proven their short-term efficacy in these patients, ${ }^{10,11}$ though there is no long-term data that show the patient's evolution in relation to pain control. Due to all this, CPN is reserved for pain control only in confirmed unresectable neoplasms.

CPB has also been evaluated in pancreatic cancer pain treatment but, because it's effect seems to be transitory and of less magnitude, it is not advised as an elective technic in oncologic pain. Its use has been proposed though in chronic pancreatitis pain treatment. Although the factors that influence chronic pancreatic pain are many, ${ }^{12}$ it's true that there is a neuropathic component that is often difficult to control with conventional analgesic drugs. As opposed to what happens with CPN, there are not many studies that describe CPB's efficacy in its different approaches, and those that exist have few patients included. In these, there is a pain-relief rate of $50 \%$ to $60 \%$, with a duration that ranges between 2 and 3 months. Re-appearance of pain is the rule after the $3^{\text {rd }}$ month and analgesia maintenance is exceptional. ${ }^{13}$ These results are equivalent to those obtained in endoscopic ultrasonography (USE) guided CPB, as we will later see.

\section{EUS technics}

Both CPN and CPB initially were EUS-guided in the 90's, according to the first description of the technic made by Wiersema et al. ${ }^{14}$ The relatively simple identification of the area where the celiac plexus is located, and the scarcely complicated technic in relation to other EUS-guided interventions, have facilitated its expansion and multiplied the publications referring to its efficacy and safety. As we will see, the results of both technics are similar or slightly better than those described in previous literature, suggesting a higher safety of the EUS-guided technic, probably due to the anterior approach and the lack of susceptible tissues between the puncture area and the celiac plexus. Both technics are done in the same way, identifying the theoretical location of the celiac plexus with a therapeutic linear ecoendoscope. From the upper portion of the gastric body, the descending aorta and the celiac trunk base are easily identified. The space located between the gastric wall, the aorta and the celiac trunk, is where the celiac plexus should be placed, and thus where we should practice the injection. ${ }^{15,16}$ It will be done with a conventional FNA needle of a variable caliber, being $20 \mathrm{G}$ and $22 \mathrm{G}$ the most commonly used. The only difference between EUS-CPN and EUS-CPB is the substance injected in the plexus. In CPN, $5 \mathrm{ml}$ of $0,25 \%$ bupivacaine are initially injected, followed by $20 \mathrm{ml}$ of absolute alcohol. The absolute alcohol volume has been evaluated in some studies, ${ }^{17}$ concluding that the $20 \mathrm{ml}$ injection in comparison to $10 \mathrm{ml}$, doesn't increase the complications or side effects, with a mild increase of its efficacy. In CPB a mixture of $20 \mathrm{ml} 0,25 \%$ bupivacaine and $80 \mathrm{mg}$ of triamcinolone is injected, usually in two successive and equivalent bolus to facilitate the flow through the interior of the needle. Although there recently has been published a study that concludes that the injection of the bupivacaine-triamcinolone mix does not cause a greater pain relief than bupivacaine on its own, ${ }^{18}$ the use of both is still the standard technic whilst waiting for more studies that confirm these results.

The injection point location in relation to the celiac plexus has also been a controversial issue. It has been suggested that the injection in both sides of the celiac trunk (bilateral technic) might facilitate a better diffusion of the applied substance in the whole plexus, in contrast with the classical o central technic. ${ }^{11}$ Available published data are controversial in relation to efficacy, ${ }^{19-21}$ but don't show differences between the technics' complications. It has recently been confirmed the possibility to visualize the celiac ganglia by EUS in a large portion of patients, ${ }^{22}$ which would imply a more precise therapeutic target with greater efficacy expectancy in pain control. As we will later see the results are promising, but there are still few studies that evaluate this fact. Complications of both technics are mild in most cases. ${ }^{23,24}$ Although initially the risk of severe complication was assumed to be similar to that described in percutaneous technics, specific studies 
of EUS reflect fewer and milder complications. The most frequent, like in percutaneous technics, are diarrhea and hypotension, probably secondary to the increase of parasympathetic activity after puncture. Both are usually mild and transitory. The frequency of each one of them is estimated to be approximately $10 \%$, though some studies describe it happening in up to $40 \%$ of the patients, especially in the case of diarrhea.

Post-puncture abdominal pain or exacerbation of the previous pain is also frequent and usually self-limited ( $9 \%$ ). A severe but exceptional $\mathrm{CPB}$ complication is the induction of retroperitoneal abscesses in the puncture area, probably secondary to bacterial translocation. Other vascular severe complications have been published as clinical cases, including a case of gastric ischemia, one case of medulla and two of hepato-splenic infarctions. ${ }^{25-28}$ Ischemic complications, which can be fatal, are considered the most serious adverse events. Four cases of acute paraplegia have been reported; in all four cases, the paraplegia was permanent. Paraplegia following EUS-guided neurolysis is thought to be caused by acute spinal cord ischemia resulting from injury to the anterior radicular artery or from vasospasm. There is a case of acute respiratory failure resulting from bilateral diaphragmatic paralysis following EUS-CPN described recently. ${ }^{29}$ In any case, these technics seem to have a lower global complication risk in comparison to those practiced through other different approaches, though it must not be forgotten that notified complications wit percutaneous technics can also happen in USE.

\section{EUS-guided CPN}

There are many studies that confirm the efficacy of EUS-guided CPN in oncologic pain treatment, mainly of pancreatic origin, though also of other etiologies. Variability amongst studies though, makes global evaluation of the efficacy and comparison with other technics more difficult. EUS-guided CPN's global efficacy has been confirmed in several meta-analyses, proving an improvement in QOL and pain evaluation scales. ${ }^{4,6,7,11}$ Most of these studies confirm a pain improvement period that varies between 2 and 3 months, ${ }^{30,31}$ decreasing its efficacy after the third month. $70 \%$ to $90 \%$ of the patients experiment post-procedure pain relief according to publishing. It is not clear though that CPN allows a decrease of opioid doses or their side effects in a statistically significant manner, since the existing results are contradictory. ${ }^{6,8,32}$ In any case, it seems clear that there is a decrease in opioid requirements at least during the first weeks, which is not statistically significant, increasing again during the final phases of the disease. The existence of multiple factors implicated in pain genesis, as well as the multiple treatments administered previous to EUS-CPN, could determine the response variability. Patients' complexity and the prioritization of QOL improvement as an objective, make a careful selection of cases necessary in which a greater efficacy is expected, to avoid unnecessary or ineffective invasive technics. Sadly it's still complicated to determine in which patients' pain response is going to be optimal. Some studies have evaluated the factors that might determine a worse pain response after $\mathrm{CPN}$, concluding that celiac plexus' local invasion can predict a poor treatment response. ${ }^{33}$ Pancreatic gland localization of the tumor can also determine a worse response to treatment, ${ }^{34}$ although in regard to this issue results are heterogeneous or even contrary when analyzing different studies' results.
The most optimal moment to apply EUS-CPN has also been evaluated. Although it has been suggested that early use of the technic might have a benefit in pain control, other studies affirm the opposite. Arm et al..$^{35}$ obtain better results in pain control and opioid doses decrease in patients that received percutaneous CPN after a conventional analgesic treatment in comparison to those that received CPN initially. Wyse et al, though, carried through a randomized and prospective specific EUS-CPN study, in which they proved a moderate decrease of pain and a opiates doses stabilization in those patients in which EUS-CPN was early applied (at the time of the histologic diagnosis) in comparison to those in which the traditional protocol was followed. ${ }^{36}$ However, it's still to be determined if palliative radiotherapy received by many of these patients might induce a bias in the results interpretation. Repeated procedures don't seem to increase efficacy, showing an important response loss after the first session. ${ }^{37}$ Nonetheless this result is referred to percutaneous technic, and might not be extrapolated to EUS-CPN. There aren't currently similar EUS studies that can endorse these results. USE-CPN complications have been briefly summarized previously, being mild and transitory in most cases. Some studies suggest that the transitory post-puncture pain exacerbation, cataloged as a mild complication, could predict a better pain response later.

\section{EUS-guided celiac ganglia neurolysis (CGN)}

As it was previously indicated, there exists the possibility of visualizing the celiac ganglia through EUS in more than $80 \%$ of the patients, regardless of the existing pathology. Direct alcohol injection in its interior would cause a more efficient destruction of the nerve conduction and thus a greater pain relief. Based on this hypothesis Levi et al carried out a preliminary retrospective study with 36 patients, in which puncture of ganglia's efficacy and safety was evaluated. ${ }^{38}$ Later, a randomized study that compared CGN and CPN proved a patent superiority of EUS-CGN over EUS-CPN, ${ }^{39}$ at the expense of EUSCPN's poor results, much worse than those previously described. The authors ascribe this to the not-differentiation of both technics, which would increase EUS-CPN's global efficacy by including a large proportion of unnoticed EUS-CGNs in such studies. In any case, direct puncture of the celiac ganglia seems to be a positive response predictor factor ${ }^{40}$ though more studies are necessary to confirm these good results.

\section{EUS-guided CPB}

Unlike EUS-CPN, there are not many studies that evaluate EUSguided CPB's efficacy. Although it was initially used in oncologic pain treatment, the higher efficacy of absolute alcohol neurolysis has reduced its indication to pain control in benign pathology, mainly chronic pancreatitis. Although there isn't a strict contraindication for EUS-CPN use in chronic pancreatitis, it seems to associate a higher complication rate than EUS-CPB, including the possibility to induce retro-peritoneal fibrosis that could interfere with the disease's course or even worsening previous pain.

As we have already seen the technic is the same as in EUS-CPN, injecting bupivacaine and triamcinolone at the mentioned doses. In $50-60 \%$ of the cases there is pain relief in an 8 -week period, progressively decreasing until the $24^{\text {th }}$ post-procedure week, being few the patients thatmaintain this effect after that time. ${ }^{41}$ These results are slightly better than those achieved with the posterior approach 
radiologic technic ${ }^{42,43}$ although they don't allow the withdrawal of analgesic medication in any of the cases, thus being its use always concomitant. The safety profile is acceptable. In fact, up to the date there haven't been registered any neurological side effects secondary to EUS-CPB. The most frequent side effects are orthostatic hypotension that responds to fluid therapy and transitory diarrhea on the first post-procedure days. Both are catalogued as mild and are usually well tolerated by the patients. Some case of post-puncture intra-abdominal abscess has been described, resolved with wide spectrum antibiotic therapy. ${ }^{23,42}$ Although it is not regularly used, this potential complication makes antibiotic prophylaxis advisable when practicing EUS-CPB. It does not seem possible to generalize EUS$\mathrm{CPB}$ in all patients with chronic pancreatitis' pain. In this pathology probably exist other more efficient therapeutic alternatives depending on the pain's etiology (e.g. lithiasis, pancreatic duct stenosis); besides, the greater life expectancy of these patients make it necessary to apply a treatment with a longer term pain control. Nevertheless, EUS-CPB could be an efficient alternative in patients with severe side effects secondary to analgesic drugs, allowing a reduction of their doses, especially in exacerbations. The factors that predict a poor response, according to some studies, would be previous pancreatic surgery and age, having worse response patients under 45 years old. ${ }^{42}$

\section{EUS-guided celiac ganglia block (CGB)}

There are no prospective studies that evaluate the efficacy of the celiac EUS-CGB. The results published by Levy ${ }^{38}$ are not as favorable as those of the EUS-CGN, neither in chronic pancreatitis nor pancreatic adenocarcinoma. In fact, they suggest a lower efficacy than that obtained with standard EUS-CPB technic (38\% vs. 55\%), maybe due to a small sample size. In any case it is not possible to advice this technic with the available information.

\section{Discussion}

EUS-CPN has proved an efficacy at least equivalent to that of percutaneous CPN, with less technic derived complications. It is notable the lack of neurological complications that have been notified since its practice started almost 20 years ago. This allows it to be considered a real alternative to percutaneous technics in pancreatic cancer pain control. Data suggest that EUS-CPN should even be considered as the elective procedure in centers where it is available. Nonetheless, in spite of its efficacy in decreasing pain intensity, it doesn't allow the interruption of opioid treatment, leaving it as adjuvant therapy. For this reason it is necessary to investigate factors that may allow to increase its efficacy, and predictive factors that might select patients in which response could be optimal. Direct EUSCGN results seem promising, and it is expected that in the future this will be the choice procedure. It seems logical that the direct injection over this structure is more effective inhibiting neuropathic routes, and available studies guarantee so. We think an extra effort will be worth making to locate the celiac ganglia in all patients submitted to apply EUS-CPN, and try its puncture in all cases. More studies are necessary to evaluate the efficacy of such intervention, but because it doesn't significantly modify the technic and has proven its safeness, it should be a real target in all the practiced procedures.

The timing in which EUS-CPN is performed is a modifiable factor that hasn't been sufficiently explored. Classically, oncologic pain in pancreatic cancer starts with oral drugs in according to WHO's analgesic ladder, frequently being necessary to use high opioid doses to achieve pain free periods. This is an important issue, since opioid side effects limit the QOL of these patients, often already deteriorated due to their underlying disease. Invasive technics have been used since they appeared only as a rescue treatment when there is no acceptable response to oral drugs, probably because even though the frequency of severe complications is low, these can be irreversible. We believe a change in this approach is necessary. The low rate of severe complications up to the date in EUS-CPN use allows it to be posed even as an initial alternative to oral analgesia. There is only one study that has evaluated EUS-CPN efficacy when early used, and the results are modest but encouraging. Studies that include only percutaneous technics we believe cannot be extrapolated to EUS. We also believe that more protocolized and EUS-CPN exclusivelycentered studies are needed to assess the ideal moment in which it should be executed. The fact that it can be carried out at the time of the cytological diagnosis, during the FNA procedure, can involve an economic saving that should be evaluated too. Currently most patients with pancreatic adenocarcinoma with unrepeatability criteria are submitted for histologic confirmation via EUS-FNA, with the intention to optimize chemotherapy. Most of these patients will later require analgesia for pain control during the disease's course. Early EUS-CPN could delay analgesic climbing and increase the opioidfree time, reducing its side effects. Wyse et als work suggests this possibility, although it hasn't proven a significant reduction of opioid doses. In any case, a better pain control as an adjuvant is not a minor target. In summary, the accomplishment of more studies in this direction, both in efficacy and cost effectiveness, seem to us a priority to optimize the technics results.

Regarding EUS-CPB, in spite that the first studies describing its role in secondary pain in chronic pancreatitis seemed promising, there is currently no evidence to recommend it as a first choice treatment. This pathology's complexity and the multifactorial etiology of its pain make EUS-CPB's results in pain control in these patients modest. It should be pointed out that CPB does not pursue the permanent ablation of the celiac plexus, and thus the reappearance of pain after a few weeks is the norm. EUS-CGB hasn't demonstrated being as efficient as EUS-CGN either, and therefore direct puncture of the celiac ganglia a priori doesn't improve results according to available data. Optimization of EUS-CPB probably first requires the adequate selection of patients with good response predictive factors. Few studies evaluate these factors, and it is not possible to determine in which patients we will obtain a better response. It's suggestive that it will be poor in patients under 45 -years old or with previous pancreatic surgery, so it's advised to try alternative treatments in these cases. In the same way, EUS-CPB use in exacerbations hasn't been sufficiently assessed, and more studies are necessary to evaluate its safety. Repeated injections don't seem to increase the efficacy and could be related to higher complication rates, so this procedure would not be a good alternative.

EUS-CPN use in chronic pancreatitis is not currently advised. Classic studies do not recommend it due to potentially severe complications (especially related to percutaneous CPN) in contrast to CPB. Nevertheless there are published cases in different series and prospective studies that suggest a better response of pain in opposition to CPB. Because this wasn't the target of these papers, those data weren't analyzed and there are no specific safety studies for this type of patients. It's obvious that absolute alcohol's lyses potential implies a more effective pain decrease at the expense of nervous endings' 
destruction. The medium and long term evolution that these patients could have, and deferred complications could develop after CPN, are unknown. For this reason its performance should be limited to selected cases in which other treatments have failed or where pain is resistant or crippling. More studies would be necessary to evaluate EUS-CPN's safeness in patients with chronic pancreatitis to be able to establish if it should be advised or counter advised. ${ }^{44-46}$

\section{Conclusion}

a. EUS-CPN is a well-established technic in management of pancreatic pain, particularly in pancreatic cancer, showing similar or even better results in pain relief than percutaneous CPN with less severe complications.

b. The timing in which EUS-CPN is performed should be closely evaluated, because it seems to be a promising factor in order to improve efficacy or even cost-effectiveness of this technic.

c. EUS-CGN should be elective as possible in patients referred to EUS-CPN, because it seems to be more effective than conventional EUS-CPN in achieving pain relief, and ganglia can be visualized in most of patients.

d. EUS-CPB or EUS-CGB are not recommended techniques in chronic pancreatitis pain, at least at first. More studies are needed to define their role in treatment of chronic pain, probably focused in patient's selection.

\section{Acknowledgement}

None.

\section{Conflict of interest}

No conflict of interest is declared.

\section{References}

1. Kappis M. Erfahrungen mit local Anasthesie bie Bauchoperationen Vehr Dtsch Gesellsch Chir. 1914;43:87-89.

2. Koizuka S, Nakajima K, Mieda R. CT-guided nerve block: a review of the features of CT fluoroscopic guidance for nerve blocks. $J$ Anesth. 2014;28(1):94-101.

3. Lillemoe KD, Cameron JL, Kaufman HS, et al. Chemical splanchnicectomy in patients with unresectable pancreatic cancer: A prospective randomized trial. Ann Surg. 1993;217(5):456-457.

4. Eisenberg E, Carr DB, Chalmers TC. Neurolytic celiac plexus block for treatment of cancer pain: a meta-analysis. Anesth Analg. 1995;80(2):290-295.

5. Kawamata M, Ishitani K, Ishikawa K, et al. Comparison between celiac plexus block and morphine treatment on quality of life in patients with pancreatic cancer pain. Pain. 1996;64(3):597-602.

6. Wong GY, Schroeder DR, Carns PE, et al. Effect of neurolytic celiac plexus block on pain relief, quality of life, and survival in patients with unresectable pancreatic cancer: a randomized controlled trial. JAMA. 2004;291(9):1092-1099.

7. Wa Zhong, Zhong Yu, Jing-Xian Zeng, et al. Celiac Plexus Block for treatment of pain associated with pancreatic cancer: A MetaAnalysis. Pain Pract. 2014;14(1):43-51.
8. Yan BM, Myers RP. Neurolytic celiac plexus block for pain control in unresectable pancreatic cancer. Am $J$ Gastroenterol. 2007;102(2):430-438.

9. Fujii-Lau LL, Bamlet WR, Eldrige JS, et al. Impact of celiac neurolysis on survival in patients with pancreatic cancer. Gastrointest Endosc. 2015;82(1):46-56.

10. Lavu H, Lengel HB, Sell NM, Baiocco JA, et al. A prospective, randomized, Double-Blind-Placebo controlled trial on the efficacy of ethanol celiac plexus neurolysis in patients with operable pancreatic and periampullary adenocarcinoma. $J$ Am Coll Surg. 2015;220(4):497-508.

11. Puli SR, Reddy JB, Bechtold ML, et al. EUS-guided celiac plexus neurolysis for pain due to chronic pancreatitis or pancreatic cancer pain: a meta-analysis and systematic review. Dig Dis Sci. 2009;54(11):2330-2337.

12. Chauhan S, Forsmark C. Pain management in chronic pancreatitis: A treatment algorithm. Best Pract Res Clin Gastroenterol. 2010; 24(3):323-335.

13. Pap A, Nauss LA, Di Magno EP. Is percutaneous celiac plexus block PCPB associated with pain relief in chronic pancreatitis? A comparison among analgesic, alcohol and steroid PCPB. Pancreas. 1990;5:725.

14. Wiersema MJ, Wiersema LM. Endosonography-guided celiac plexus neurolysis. Gastrointest Endosc. 1996;44(6):656-662.

15. Collins D, Penman I, Mishra G, et al. EUS-guided celiac block and neurolysis. Endoscopy. 2006;38(9):935-939.

16. Penman ID, Gilbert D. Basic technique for celiac plexus block/ neurolysis. Gastrointest Endosc. 2009;69(2 Suppl):S163-165.

17. LeBlanc JK, Rawl S, Juan M, et al. Endoscopic Ultrasound-Guided Celiac Plexus Neurolysis in pancreatic cancer: A Prospective Pilot Study of safety using $10 \mathrm{~mL}$ versus $20 \mathrm{~mL}$ alcohol. Diagn Ther Endosc. 2013;2013:327036.

18. Stevens T, Constanzo A, Lopez R, et al. Adding Triamcinolone to Endoscopic Ultrasound-Guided Celiac Plexus Blockade does not reduce pain in patients with chronic pancreatitis. Clin Gastroenterol Hepatol. 2012;10(2):186-191.

19. Sahai AV, Lemelin V, Lam E, et al. Central vs. bilateral endoscopic ultrasound-guided celiac plexus block or neurolysis: a comparative study of short-term effectiveness. Am J Gastroenterol. 2009;104(2):326-329.

20. LeBlanc J, Al-Haddad M, McHenry L, et al. A prospective, randomized study of EUS-guided celiac plexus neurolysis for pancreatic cancer: one injection or two?. Gastrointest Endosc. 2011;74(6):1300-1307.

21. LeBlanc JK, DeWitt J, Johnson C, et al. A prospective randomized trial of 1 versus 2 injections during EUS-guided celiac plexus block for chronic pancreatitis pain. Gastrointest Endosc. 2009;69(4):835842 .

22. Levy M, Rajan E, Keeney G, et al. Neural ganglia visualized by endoscopic ultrasound. Am J Gastroenterol. 2006;101(8):1787-1791.

23. O’Toole TM, Schmulewitz N. Complication rates of EUS-guided celiac plexus blockade and neurolysis: results of a large case series. Endoscopy. 2009;41(7):593-597.

24. Adler DG, Jacobson BC, Davila RE et al. ASGE guideline: complications of EUS. Gastrointest Endosc. 2005;61(1):8-12. 
25. Gimeno-García AZ, Elwassief A, Paquin SC, et al. Fata complication after EUS-guided celiac plexus neurolysis. Endoscopy. 2012;44(suppl 2):E267.

26. Loeve US, Mortensen MB. Lethal necrosis and perforation of the stomach and the aorta after multiple EUS-guided celiac plexus neurolysis procedures in a patient with chronic pancreatitis. Gastrointest Endosc. 2013;77(1):151-152.

27. Fujii L, Clain JL, Morris JM, et al. Anterior spinal cord infarction with permanent paralysis following endoscopic ultrasound celiac plexus neurolysis. Endoscopy. 2012;44:E265-E266.

28. Jang HY, Cha SW, Lee BH, et al. Hepatic and splenic infarction and bowel ischemia following endoscopic ultrasound-guided celiac plexus neurolysis. Clin Endosc. 2013;46(3):306-309.

29. Minaga K, Takenaka M, Kamata K, et al. Alleviating Pancreatic Cancer-Associated Pain Using Endoscopic Ultrasound-Guided Neurolysis. Cancers (Basel). 2018;10(2):50.

30. Seicean A, Cainap C, Gulei I, et al. Pain palliation by endoscopic ultrasound-guided celiac plexus neurolysis in patients with unresectable pancreatic cancer. $J$ Gastrointestin Liver Dis. 2013;22(1):59-64.

31. Wiechowska-Kozłowska A, Boer K, Wojcicki M, et al. The efficacy and safety of endoscopic ultrasound-guided celiac plexus neurolysis for treatment of pain in patients with pancreatic cancer. Gastroenterol Res Pract. 2012;2012:503098.

32. Gunaratnam NT, Sarma AV, Norton ID, et al. A prospective study of EUS-guided celiac plexus neurolysis for pancreatic cancer pain. Gastrointest Endosc. 2001;54(3):316-324.

33. Iwata K, Yasuda I, Enya M, et al. Predictive factors for pain relief after Endoscopic Ultrasound-guided celiac plexus neurolysis. Dig Endosc. 2011;23(2):140-145.

34. Rykowski JJ, Hilgier M. Efficacy of neurolytic celiac plexus block in varying locations of pancreatic cancer: influence on pain relief Anesthesiology. 2000;92(2):347-354.

35. Amr YM, Makharita MY. Comparative study between 2 protocols for management of severe pain in patients with unresectable pancreatic cancer: one-year follow-up. Clin J Pain. 2013;29(9):807-813.

36. Wyse JM, Carone M, Paquin SC, et al. Randomized, double-blind, controlled trial of early endoscopic ultrasound-guided celiac plexus neurolysis to prevent pain progression in patients with newly diagnosed, painful, inoperable pancreatic cancer. $J$ Clin Oncol. 2011;29(26):3541-3546.

37. McGreevy K, Hurley RW, Erdek MA, et al. The effectiveness of repeat celiac plexus neurolysis for pancreatic cancer: a pilot study. Pain Pract. 2013;13(2):89-95.

38. Levy MJ, Topazian MD, Wiersema MJ, et al. Initial evaluation of the efficacy and safety of endoscopic ultrasound-guided direct Ganglia neurolysis and block. Am J Gastroenterol. 2008;103(1):98-103.

39. Doi S, Yasuda I, Kawakami H, et al. Endoscopic ultrasound-guided celiac ganglia neurolysis vs. celiac plexus neurolysis: a randomized multicenter trial. Endoscopy. 2013;45(5):362-369.

40. Ascunce G, Ribeiro A, Reis I, et al. EUS visualization and direct celiac ganglia neurolysis predicts better pain relief in patients with pancreatic malignancy. Gastrointest Endosc. 2011;73(2):267-274.

41. Gress F, Schmitt C, Sherman S, et al. Endoscopic ultrasound-guided celiac plexus block for managing abdominal pain associated with chronic pancreatitis: a prospective single center experience. $\mathrm{Am} J$ Gastroenterol. 2001;96(2):409-416.

42. Gress F, Schmitt C, Sherman S, et al. A prospective randomized comparison of endoscopic ultrasound and computed tomographyguided celiac plexus block for managing chronic pancreatitis pain. Am J Gastroenterol. 1999;94(4):900-905.

43. Santosh D, Lakhtakia S, Gupta R, et al. Clinical trial: a randomized trial comparing fluoroscopy guided percutaneous technique vs. endoscopic ultrasound guided technique of coeliac plexus block for treatment of pain in chronic pancreatitis. Aliment Pharmacol Ther. 2009;29(9):979-984.

44. Levy MJ, Chari ST, Wiersema MJ. Endoscopic ultrasound-guided celiac neurolysis. Gastrointest Endosc Clin N Am. 2012;22(2):231247.

45. Kaufman M, Singh G, Das S, et al. Efficacy of endoscopic ultrasoundguided celiac plexus block and celiac plexus neurolysis for managing abdominal pain associated with chronic pancreatitis and pancreatic cancer. J Clin Gastroenterol. 2010;44(2):127-134.

46. Seicean A. Celiac plexus neurolysis in pancreatic cancer: the endoscopic ultrasound approach. World $J$ Gastroenterol. 2014;20(1):110-117. 